\title{
Estimación del descarte de material reciclable doméstico en Chiriquí, Panamá
}

\section{Estimation of the disposal of domestic recyclable material in Chiriqui, Panama}

\author{
Mónica Aparicio ${ }^{1}$, Conny Hernández ${ }^{1}$, Lidiet. Fossatti ${ }^{1}$, Aylin Castillo ${ }^{1}$, Jorge Luis Pino ${ }^{2 *}$ \\ ${ }^{1}$ Departamento de Biología, Facultad de Ciencias Naturales y Exactas, Universidad Autónoma de Chiriquí, \\ ${ }^{2}$ Vicerrectoría de Investigación y Postgrado,Universidad Autónoma de Chiriquí
}

\begin{abstract}
Resumen El manejo y disposición de residuos sólidos a nivel mundial constituye un problema; América Latina, y Panamá no escapan a esta realidad. El objetivo del presente fue es estimar la cantidad de plástico y vidrio reciclable descartado en viviendas en la provincia de Chiriquí. A su vez, se aprovechó para educar a las personas sobre los beneficios del reciclaje y el problema que representa el plástico y vidrio en nuestra población. Durante un mes se recolectaron y se pesaron los residuos de plástico y vidrio reciclable acumulados. Se produjo $26.03 \mathrm{~kg} / \mathrm{mes}$ de plástico reciclable en ocho casas muestreadas, con un promedio de $3.25 \mathrm{~kg} / \mathrm{mes}$ de descarte reciclable por casa. Solamente, una de las viviendas proporcionó insumos para estimar el descarte de vidrio reciclable; el cual fue de $1.94 \mathrm{~kg} / \mathrm{mes}$ de envases de vidrio, lo que podría indicar que las familias tienden a reutilizar más el vidrio que el plástico. Con base en la información generada, se puede estimar que en estas viviendas podrían estar produciendo unas 0.312 toneladas de plástico por año. Este trabajo representa la primera estimación publicada, sobre la generación de desechos reciclables domiciliarios en David, Chiriquí; información de pertinencia para la formulación de iniciativas de reciclaje y manejo de desechos sólidos locales.
\end{abstract}

Palabras clave Descarte de desechos, Chiriquí, plástico, reciclaje, vidrio.

\begin{abstract}
The management and disposal of solid waste is a worldwide problem; Latin America, and Panama does not escape to this reality. The goal of this work was to estimate of the amount of domiciliary recyclable plastic and glass discarded in the Province of Chiriquí. While developing the research, environmental information about benefits of recycling was presented to educate people about the problem of residual plastic and glass. During a month, the accumulated recyclable plastic and glass waste was collected and weighed. A total of $26.03 \mathrm{~kg} / \mathrm{month}$ of recyclable plastic was produced from eight sampled houses, with an average of 3.25 $\mathrm{kg} / \mathrm{month}$ of recyclable waste per house. Only one of the houses provided data to estimate the disposal of recyclable glass; which was $1.94 \mathrm{~kg} / \mathrm{month}$ of glass containers, this could indicate that families trend to reuse glass more than plastic. Based on the information generated, it can be estimated that these houses could be producing about 0.312 tons of plastic per year. This work represents the first published estimate on the production of household recyclable waste in David, Chiriquí; which could be information of relevance for the formulation of local solid waste recycling and management initiatives.
\end{abstract}

Keywords Waste disposal, Chiriquí, plastic, recycling, glass.

*Corresponding author: jorge.pino@unachi.ac.pa

\section{Introducción}

El manejo y disposición de residuos sólidos a nivel mundial constituye un problema grave y las ciudades de América Latina no escapan a esta realidad [1]. Factores como la insuficiente recolección y la inadecuada disposición final de residuos sólidos entre otros, provocan contaminación de tierra, aguas y aire, y genera riesgos para la salud humana. La Organización de las Naciones Unidas para el Desarrollo Industrial define desecho como todo lo que es generado como producto de una actividad, ya sea por la acción directa del hombre o por la actividad de otros organismos vivos, formándose una masa heterogénea que, en muchos casos, es difícil de reincorporar a los ciclos naturales [2].

Estadísticas llevadas a cabo por la Cámara de Reciclaje de Panamá han demostrado que el país genera cerca de 3 mil 800 toneladas de basura al día y solo se recicla alrededor de un 5\%, mientras que en otros países están reciclando al menos un 50\% (La Estrella de Panamá, 2017). Por lo tanto, se puede considerar que en Panamá existe la capacidad de desarrollar actividades encaminadas al reciclaje.

La basura proveniente de los hogares y/o comunidades se clasifican en: 1) envases de vidrio, 2) plástico fino, 3) plástico 
grueso, 4) cartón, 5) varios, 6) latas compactadas, 7) papel, 8) baterías, 9) metales diversos, 10) orgánicos, 11) tetrapak, 12) telas, 13) sanitarios [3].

En Panamá, un $8 \%$ de los desechos domésticos se compone de vidrio, el cual de ser reutilizado, podría desviar su destino al vertedero o como contaminante del entorno [3]. El plástico y el vidrio son de los materiales que más tardan en descomponerse, y al mismo tiempo los más utilizados en las sociedades actuales. Se calcula que el plástico tarda entre 100 y 1,000 años en descomponerse, mientras que el vidrio se degrada aproximadamente en 4,000 años [4]. Aunque en la "literatura gris" panameña se encuentran guías con descripciones de materiales reciclables para nuestro país [5], estas carecen de estimaciones locales.

Los microplásticos pequeñas partículas de plástico empleadas en una gran variedad de productos de limpieza [6] son producidos durante la degradación del plástico ya utilizado así como también durante la manufactura en muchas industrias. Debido a su pequeño tamaño (inferiores a $5 \mathrm{~mm}$ ), los microplásticos, son demasiado pequeños para ser eliminados por sistemas de filtración de aguas residuales y terminan en ríos y océanos, en donde son ingeridos por pájaros, peces y otras especies marinas. Los microplásticos, como producto del plástico doméstico no reciclado, constituyen una gran amenaza para la alimentación de animales marinos, incluidas muchas especies de aves, tortugas, peces, mamíferos marinos e invertebrados, que los confunden con alimento, provocando su muerte [6].

Chiriquí está ubicada en la región occidental de Panamá, particularmente en el Distrito de David, la provincia de Chiriquí cuenta con un total de 416,873 habitantes y 134,369 viviendas [7], y posee un sistema recolección de material de descarte, que traslada este descarte a un vertedero sin realizar un proceso de clasificación de la basura; por lo que se espera que parte de los plásticos descartados podrían llegar a representar algún problema ecológico o de salud pública. Ante la visión de disminuir el impacto de los desechos sólidos, son recurrentes los llamados a desarrollar programas de reciclaje de elementos reutilizables como lo son el vidrio y el plástico.

Para poder desarrollar iniciativas que estén relacionadas con el reciclaje de productos de descartes, es necesario realizar mediciones sobre la producción de material de descarte reciclable en la población. La finalidad de este trabajo es brindar una primera aproximación sobre el descarte de material de plástico y vidrio reciclable en familias de tamaño promedio en el distrito de David.

\section{Métodos y materiales}

\section{1 Área de estudio}

El muestreo se realizó en tres sectores de la provincia de Chiriquí, específicamente en los distritos de Dolega, Boquerón y David; las áreas guardan relación con los domicilios de los autores, lo que facilitó el desarrollo del trabajo. Los hogares muestreados se localizaron en la periferia de la ciudad de David, la cual representa la cuarta ciudad más poblada del país [7] y la más desarrollada en la Región Occidental.

\subsection{Materiales}

En la literatura se encuentran diversos métodos utlizados para estimar la producción de residuos reciclables, los cuales estos suelen ser ajustados a las características del estudio y del entorno. En este trabajo, se realizó una estimación directa del material de descarte doméstico de manera selectiva [2].

En este estudio se utilizaron bolsas negras para basura, para recolectar el material de plástico y vidrio proporcionado; y una balanza colgante para el pesaje de la misma (figura 1).

Se contactó a diez familias para ser parte del estudio. En estos hogares mantenía un tamaño familiar de entre cuatro y seis miembros. En cada casa se proporcionó material informativo impreso que describía la finalidad del estudio y con la simbología referida a los tipos de material reciclable, para que pudiesen identificar este material dentro de su descarte de basura. Se les solicitó su contribución en la separación del material reciclable de descarte y colocación en las bolsas proporcionadas para vidrio y plástico.

\subsection{Recolección del material de descarte}

El muestreo se realizó entre los meses de julio y agosto de 2018. Se recolectó de manera semanal el material proporcionado en cada hogar hasta cumplir con un mes de recolecta en cada vivienda. Este material de descarte fue verificado para asegurar que cumpliese con la condición de reciclable y posteriormente se estimó su masa en kilogramos mediante el uso de una balanza colgante (figura 2).

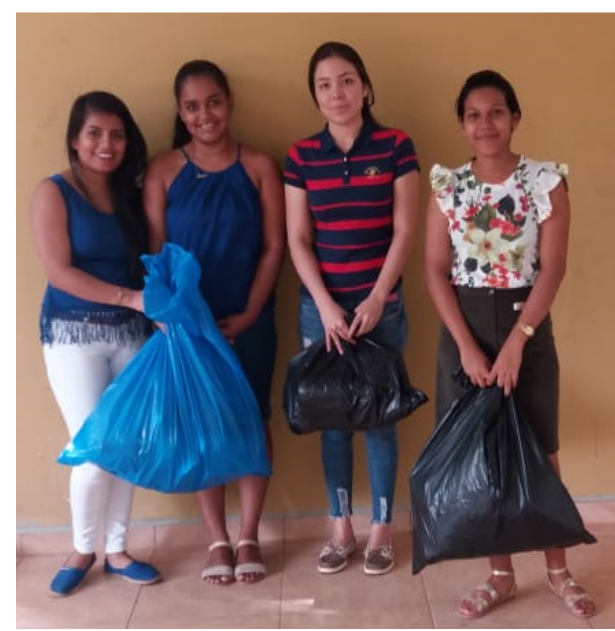

Figura 1. Recolección del material reciclable de descarte producido semanalmente en las viviendas estudiadas. 


\subsection{Organización y tabulación de datos}

Los datos obtenidos fueron tabulados y analizados, apoyados en la estadística descriptiva. Se organizaron los datos correspondientes a los pesos obtenidos en cada colecta semanal de la basura por vivienda; dichos datos se colocaron en tablas para ser comparados y posteriormente representarlos gráficamente.

\section{Resultados y discusión}

Inicialmente se contempló muestrear la producción de material de descarte reciclable en diez viviendas, pero dos de éstas fueron retiradas del estudio, ya que no cumplieron con brindar accesibilidad al material solicitado. Los resultados presentados están basados en ocho viviendas, ver tabla 1.

En Panamá, el plástico es uno de los desechos que se producen y acumulan con gran frecuencia, pero, aun así, existe un desconocimiento generalizado sobre cuánto plástico o vidrio es producido, contemplando otra unidad de referencia (por habitante, por vivienda, etc.). Por ello, los valores reportados, con el abordaje de las ocho viviendas constituye un aporte en el entendimiento de la producción estos materiales.

Los datos obtenidos nos informan sobre la generación de plástico como descarte en el sector doméstico, lo cual podría ayudar en el sustento de estrategias de estudio y manejo de elementos reciclables en el material de descarte domiciliario.

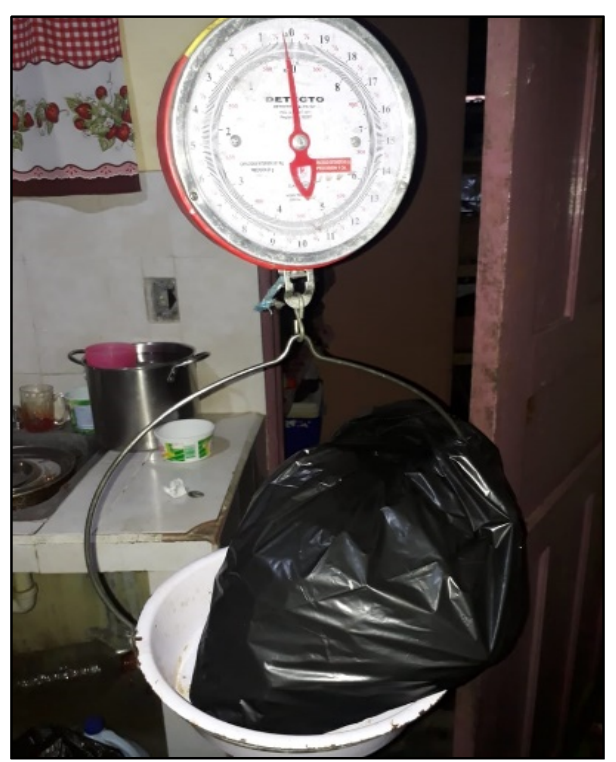

Figura 2. Pesaje semanal del material de descarte producido en cada una de las ocho viviendas.

Panamá es uno de loa países más rezagadas en el manejo y aprovechamiento de residuos en Latinoamérica [8]. Por ejemplo, uno de los problemas que enfrentamos, es que no existen los espacios para seguir depositando residuos sólidos; a su vez, los actuales vertederos generan malestar y rechazo en las comunidades. El grado de participación, directo con los participantes del estudio, permitió conocer sobre la disposición que puede tener la comunidad para involucrarse en actividades que ayuden a conocer sobre los residuos domiciliarios y para darles un mejor manejo a estos.

El abordaje utilizado para el manejo de los residuos domiciliarios no ha cambiado en décadas, se basa en la recolección y vertido de los mismos en un campo abierto, y no tanto en la reducción de su producción, reutilización, reciclaje y/o aprovechamiento energético o de compostaje de estos, que es hacia donde deberían estar enfocadas las estrategias de manejo de desechos [9].

La producción de plástico reciclable fue asimétrica por casa, variando desde $1.93 \mathrm{Kg}$ hasta $4.08 \mathrm{Kg}$ en un mes (figura 3), con un promedio de $3.25 \mathrm{Kg}$.

Tabla 1. Peso en kilogramos del plástico reciclable descartado por vivienda por semana

\begin{tabular}{|c|c|c|c|c|c|c|c|c|}
\hline & \multicolumn{7}{|c|}{ Viviendas } \\
\hline $\begin{array}{c}\text { Frecuencia } \\
\text { de } \\
\text { recolectas }\end{array}$ & V1 & V2 & V3 & V4 & V5 & V6 & V7 & V8 \\
\hline Semana 1 & 0.73 & 0.45 & 0.63 & 1.36 & 0.77 & 0.54 & 0.45 & 0.68 \\
\hline Semana 2 & 0.90 & 0.68 & 0.63 & 0.90 & 0.90 & 1.09 & 0.68 & 1.00 \\
\hline Semana 3 & 0.90 & 0.90 & 0.45 & 1.13 & 0.68 & 0.59 & 1.68 & 1.13 \\
\hline Semana 4 & 0.45 & 0.22 & 0.22 & 0.81 & 0.90 & 0.81 & 1.27 & 1.50 \\
\hline $\begin{array}{c}\text { Peso Total } \\
\text { (Kg) }\end{array}$ & $\mathbf{2 . 9 8}$ & $\mathbf{2 . 2 5}$ & $\mathbf{1 . 9 3}$ & $\mathbf{4 . 2 0}$ & $\mathbf{3 . 2 5}$ & $\mathbf{3 . 0 3}$ & $\mathbf{4 . 0 8}$ & $\mathbf{4 . 3 1}$ \\
\hline
\end{tabular}

Se muestrearon viviendas con un número de integrantes entre cuatro y seis personas; esto, para que el número de miembros de la familia no fuese la principal fuente de variación en la producción del material de descarte. Aparte de la variación reportada, también se puede notar que no hay una producción de plástico nula o muy cercana a cero. Esto podría ofrecer indicios sobre la cultura, ya sea consumista o de reciclaje (reconociendo que hay condiciones intermedias y situacionales), en la muestra estudiada. Por ejemplo, se esperaría que, si las familias hubiesen tenido un número familiar más alto, se produciría mayor cantidad de plástico reciclable; esto, en ausencia de un programa de concienciación sobre el problema de los plásticos en el ambiente.

El poder adquisitivo podría ser otro factor que potencialmente pudiese influir en la producción de plásticos como descarte. Un ejemplo que se puede mencionar es si una familia cuenta con mayor recurso económico, se le facilitaría la compra de una mayor cantidad de productos embotellados en comparación con otra familia con un presupuesto menor; tal y como ocurre a nivel mundial en donde de países con mayor poder adquisitivo son los que mayormente consumen agua embotellada [10]. 
Otros factores que podría influir en el consumo y la producción de material de plástico reciclable como descarte, podrían ser el contexto geográfico y las características culturales de la población. Por ejemplo, la temporada del año, ya que se esperaría, que en temporadas de verano se promocione más el consumo de bebidas embotelladas al igual que durante celebraciones de fin de año, o patronales regionales.

Los datos reportados sobre el descarte de materiales plásticos, permiten reflexionar sobre las dimensiones de estas cifras, ya que solo se contabilizó una fracción muy pequeña de las zonas urbanas de la provincia de Chiriquí sin tomar en consideración las zonas rurales y el resto de las provincias del país. Así, los resultados muestran que, en solo ocho viviendas, se puede obtener una producción de plástico reciclable de $26.03 \mathrm{Kg}$; lo que equivaldría a unas 0.026 toneladas por mes. Este valor puede ser transformado a una producción anual, lo cual sería unas 0.312 toneladas. Si extrapolamos el valor obtenido, a un escenario como el número de viviendas en la provincia de Chiriquí que es de 134,369 toneladas [11], considerando condiciones similares, se generarían unas 41,923.128 toneladas de plástico anuales, que podrían ser recicladas.

De acuerdo con datos de la Alcaldía de Panamá, el 19\% de los residuos que se manejan en el relleno sanitario de Cerro Patacón, el cual recibe residuos de la ciudad capital y alrededores, son plásticos; sólo los desechos orgánicos representan un porcentaje mayor (30\%) [3], [12]. Estas mismas fuentes, indican que la importación de plásticos durante el 2015, fue de 143,6 toneladas; lo que puede ejemplificar la potencial magnitud de contaminación ambiental que podría generar el plástico en sus distintas formas; pero también muestra la potencialidad de contar con plásticos reciclables

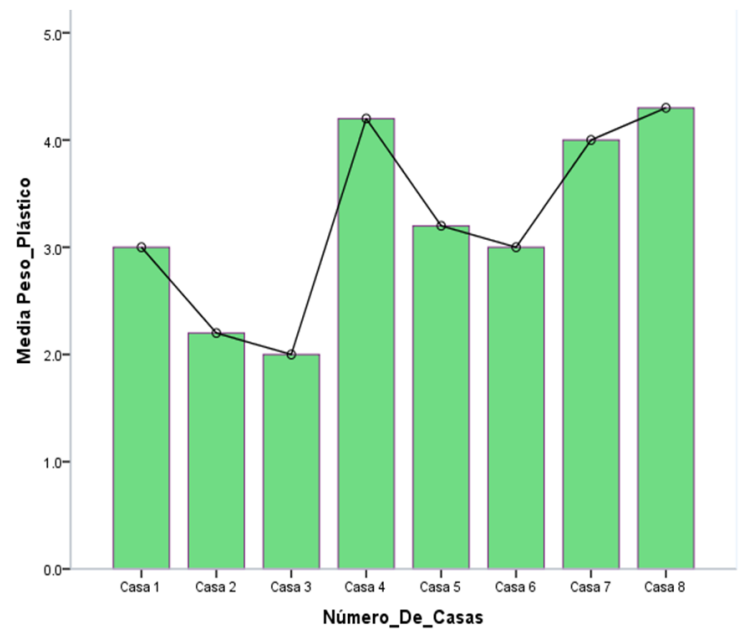

Figura 4. Variación en el peso de material plástico reciclable en kilogramos producido por vivienda muestreada.
El gran problema del plástico es intrínseco a su utilidad; por ejemplo, la mayoría de los productos fabricados con este material tienen una vida útil muy corta y suelen ser desechados con facilidad. Sin embargo, a pesar de la resistencia del plástico con características reciclables o re-usables, este. generalmente no se le potencia su re-uso. Como consecuencia, el plástico ha proliferado en nuestro entorno, desde la envoltura de los alimentos, la ropa, hasta en la mayoría de los dispositivos electrónicos que usamos.

La manera más común de eliminar los plásticos es mediante un proceso de descomposición conocido como pirólisis [13], o a través de la incineración, aunque este último procedimiento no es recomendado por sus efectos nocivos para la salud y al medio ambiente [14]. Recientemente se han descubierto otras formas de biodegradación a través de varios organismos [15]. Cualquiera que sea el método de tratamiento del plástico postdescarte, su implementación requiere la caracterización y estimación del mismo a nivel local.

Una ventaja del plástico es que, al reciclarse, podría mantenerse en uso. Una botella de agua, por ejemplo, puede reusarse hasta 20 veces sin que pierdan las propiedades del material, aunque algunos autores indican que podrían usarse por más tiempo. Sin embargo la mayoría de los residuos de plástico reciclables que existen hoy en la naturaleza, muy probablemente no fueron reciclados [16].

Actualmente en Panamá, se puede apreciar en la población, un incremento en el respaldo a iniciativas de reciclaje, los cuales son cada vez más evidentes a través de diferentes redes sociales. Un ejemplo de esta positiva receptividad, está representada por el apoyo recibido durante la realización de este proyecto a través de la información que nos fue provista en ocho de las diez viviendas (80\%).

En cuanto a la producción de desechos de vidrio reciclable, este resultó tener el manejo más ecológico por sus cualidades reciclables y rendimientos múltiples; por ejemplo, su reutilización revierte en beneficios directos inmediatos. Solamente en una de las diez viviendas contempladas en el estudio se brindó información relacionada con la producción de vidrio reciclable. La cantidad obtenida durante el mes en estudio fue de $1.94 \mathrm{Kg}$ de vidrio.

Algunas de las explicaciones brindadas sobre el descarte de los materiales de vidrio reciclables, tienen que ver con su reutilización inmediata en la cocina para guardar alimentos, para guardar monedas o como floreros; por lo que no son descartados después de su uso inicial. Otro factor que podría causar el bajo reporte de vidrios reciclables podría ser el hecho de que muchos alimentos ahora tienen una presentación en envases tetrapak, o en envases de sobres de aluminio, lo que reduce el número de productos envasados en vidrio. En este estudio se pudo evidenciar la reutilización más efectiva del vidrio sobre los plásticos. 
En el entorno industrial y comercial, el reciclaje de envases de vidrio podría formar parte de las estrategias de negocio, beneficiando a las empresas más allá de cumplir con aspectos relacionados con la responsabilidad social; promoviendo a la producción verde para que sea económicamente más atractiva.

El reciclado de vidrio generado en hogares, es un tema que requiere de mayor atención por parte de las autoridades nacionales. Contrastando con los adelantos tecnológicos y el desarrollo económico-empresarial; Panamá ha sido uno de los países en donde ha costado desarrollar programas efectivos de reciclaje y manejo de desechos. Mientras, unos ven el vidrio como un problema, empresarios latinoamericanos dedicados al reciclaje de vidrio en América Latina y el Caribe del Grupo, encuentran en vidrio una fuente importante de negocio ecoamigable.

Diferentes autores sugieren que en el reciclaje de vidrio utiliza 26\% menos de energía que la energía utilizada en la producción original del mismo. Para tener una mejor idea, en la generación de un kilo de vidrio se necesitan unas 4.200 kilocalorías de energía [17]. Al utilizar menos energía en la generación de material a través del reciclado, esto reduciría en un 20\% la contaminación atmosférica (gases de efecto invernadero), disminuyendo también la contaminación de agua en un $40 \%$ [18]; esto, aparte del ahorro equivalente del material para la producción del vidrio "virgen".

Los valores de materiales reciclables de descarte (plástico y vidrio) generados en este estudio podrían ser de interés para las autoridades municipales locales, ya que brinda valores no teóricos, sino reales sobre el descarte en viviendas, esto podría ser de utilidad para gestionar iniciativas de manejo ante el creciente problema de la basura. También podría ser de interés para empresarios interesados en desarrollar la industria del reciclaje o bien del tratamiento general de desechos sólidos.

Un ejemplo emergente para el desarrollo de industrias relacionadas con el reciclaje de plástico, surge ante la reciente necesidad mundial, debido a la pandemia, de producir masivamente máscaras de protección con pantalla de acrílico, para evitar el contagio de Covid-19. Como resultado, múltiples instituciones locales e internacionales implementaron la impresión 3D de las piezas de plástico de estas máscaras. Tal vez estas piezas podrían ser impresas con material reciclado, utilizando protocolos adecuados, ya publicados para el uso de plástico reciclado en la impresión 3D [19].

La generación de desechos sólidos reciclables puede ser heterogénea en una comunidad [20]; por ejemplo, algunos factores que podrían influir en estas variaciones se pueden mencionar factores como los ritmos de crecimiento poblacional, la distribución asimétrica de los habitantes en un contexto geográfico, o los hábitos de consumo. En este trabajo reconocemos la baja representatividad temporal y espacial del muestreo, lo cual podría ser un argumento válido para repensar en la generalización presentada a partir de los datos obtenidos. Sin embargo, en ausencia de otro tipo de información generada referente a la producción de desechos reciclables en viviendas de la provincia de Chiriquí, nuestros resultados podrían utilizarse como hipótesis para futuros trabajos.

Este es uno de los primeros estudios que genera este tipo de información en viviendas de Chiriquí. La información presentada podría ser útil en la toma de decisiones referentes a estrategias de manejo de este tipo de materiales, en el desarrollo de estrategias de concienciación sobre la gran cantidad de material de descarte producido, también incentiva a buscar soluciones creativas para el tratamiento de estos materiales con incentivos económicos, principalmente el plástico, cuya proliferación continúa afectando a la fauna y flora en ecosistemas marinos.

\section{Conclusiones}

- A través del trabajo realizado se pudo generar información base sobre la producción de vidrio y plástico reciclable en viviendas de la provincia de Chiriquí.

- El diseño empleado para la toma de datos es sencillo, confiable y escalable.

- Los valores obtenidos en este estudio presentan una importancia local para las autoridades municipales y para potenciales empresarios en el negocio del reciclaje.

\section{AGRADECIMIENTOS}

Extendemos nuestro agradecimiento las familias que participaron en esta investigación. También a la Jornada de Iniciación Científica (JIC), en cuyo marco se realizó este trabajo.

\section{REFERENCIAS}

[1] L. Á. Rodríguez Escobar, "Hacia la gestión ambiental de residuos sólidos en las metrópolis de América Latina," Rev. Innovar J. Rev. Ciencias Adm. y Soc., vol. 20, 2002, doi: 10.1017/CBO9781107415324.004.

[2] E. Rondón Toro, M. Szantó Narea, J. F. Pacheco, E. Contreras, and A. Gálvez, Guía general para la gestión de residuos sólidos domiciliarios. Santiago: CEPAL -Naciones Unidas, 2016.

[3] Defensoría del Pueblo de la República de Panamá, Informe especial sobre el manejo de los residuos sólidos en Panamá. Panamá: Gobierno Nacional, 2007.

[4] C. Sanz Boix, M. D. Salvador Moya, F. Segovia López, V. Amigó Borrás, and A. T. Pérez Rodríguez, "Degradación química de plásticos reforzados en medios básicos," VIII Congr. Nac. Propiedades Mecánicas Sólidos, pp. 341-347, 2002.

[5] "Guía de reciclaje de residuos sólidos domiciliarios." Centro Nacional de Información sobre Producción Más Limpia y Consumo Sustentable, Panamá.

[6] E. Huerta Lwanga, "Microplásticos: insospechado problema ambiental," Ecofronteras, vol. 20, no. 58, pp. 26-28, 2016. 
[7] Instituto Nacional de Estadística y Censo, Panamá en Cifras. Panamá: Departamento de Artes Gráficas, Dirección de Administración y Finanzas, 2018.

[8] S. Lobo, M. Marín, V. Rudin, and F. Salas, Análisis de los retos para el desarrollo de la cadena de valor del reciclaje en Centroamérica. Iniciativa Regional para el Reciclaje Inclusivo, 2016.

[9] INECO, "Plan Nacional de gestión integral de residuos 20172027. Modelo de gestión de residuos: Propuesta de nuevo modelo de gestión y del nuevo modelo económico financiero." INECO, La Habana, p. 122, 2017.

[10] C. Ferrier, "Water Bottled: Understanding a social phenomenon," AMBIO A J. Hum. Environ., vol. 30, no. 2, pp. 118-119, 2001, doi: 10.1579/0044-7447-30.2.118.

[11] INEC, Distribución territorial y migración interna en Panamá: Censo 2010. Panamá: Contraloria General de la Republica de Panama, 2014.

[12] La Estrella de Panamá, "En Panamá entusiasma el reciclaje pese a poca conciencia de peligro del plástico," 3 de Junio, Panamá, pp. 1-9, 2018.

[13] M. Mancheno, S. Astudillo, P. Arévalo, I. Malo, T. Naranjo, and J. Espinoza, "Aprovechamiento energético de residuos plásticos obteniendo combustibles líquidos, por medio de pirólisis," La Granja Rev. Ciencias la Vida, vol. 23, no. 1, pp. 5359, 2016, doi: 10.17163/lgr.n23.2016.06.
[14] N. Tangri, Incineración de residuos: una tecnología muriendo. 2005.

[15] J. Yang, Y. Yang, W. M. Wu, J. Zhao, and L. Jiang, "Evidence of polyethylene biodegradation by bacterial strains from the guts of plastic-eating waxworms," Environ. Sci. Technol., vol. 48, no. 23, pp. 13776-13784, 2014, doi: 10.1021/es504038a.

[16] S/A, 2016 United States National postconsumer plastic bottle recycling report. The Association of Plastic Recyclers \& The American Chemistry Council, 2016.

[17] M. A. González Lozano and P. Ponce Peña, "Uso de vidrio de desecho en la fabricación de ladrillos de arcilla," Rev. Iberoam. las Ciencias Biológicas y Agropecu., vol. 1, no. 2, pp. 1-14, 2012.

[18] K. Tarnocki, "Conocimiento de los alumnos ucabistas con respecto al reciclaje y ahorro de energía," Universidad Católica Andrés Bello, 2010.

[19] Herianto, S. I. Atsani, and H. Mastrisiswadi, "Recycled Polypropylene Filament for 3D Printer: Extrusion Process Parameter Optimization," IOP Conf. Ser. Mater. Sci. Eng., vol. 722, no. 1, pp. 1-7, 2020, doi: 10.1088/1757-899X/722/1/012022.

[20] A. P. Of, T. H. E. Northeast, M. Council, O. F. Governments, and W. Grant, "Recycling in Michigan: successful recycling programs, best practices, and diversion potential," no. January. Northeast Michigan Council of Governments, Michigan, p. 127 p, 2016. 